\title{
SHEAR WAVE ELASTOGRAPHY FINDINGS OF PSEUDOANGIOMATOUS STROMAL HYPERPLASIA
}

\author{
Hakan Abdullah Ozgul', Isıl Basara Akin¹, Merih Guray Durak², Ali Ibrahim \\ Sevinc $^{3}$, Suleyman Ozkan Aksoy ${ }^{3}$ Pinar Balci ${ }^{1}$
}

\author{
${ }^{1}$ Department of Radiology, Dokuz Eylul University, Faculty of Medicine, Izmir, Turkey \\ ${ }^{2}$ Department of Pathology General Surgery, Dokuz Eylul University, Faculty of Medicine, Izmir, Turkey \\ ${ }^{3}$ Department of General Surgery, Dokuz Eylul University, Faculty of Medicine, Izmir, Turkey
}

\author{
Address for Correspondence: Hakan Abdullah Ozgul, E-mail: haozgul@hotmail.com \\ Received: 05.01.2021; Accepted: 14.04.2021; Available Online Date: 27.05.2021 \\ @C Copyright 2021 by Dokuz Eylül University, Institute of Health Sciences - Available online at https://dergipark.org.tr/en/pub/jbachs
}

Cite this article as: Ozgul HA, Basara Akin I, Durak MG, Sevinc Al., Aksoy SO, Balci P, Shear wave elastography findings of tumoral pseudoangiomatous stromal hyperplasia. J Basic Clin Health Sci 2021; 2: 172-175.

\begin{abstract}
Pseudoangiomatous stromal hyperplasia (PASH) is a benign entity defined in 1987. Conventional breast imaging methods, including mammography, ultrasonography, and magnetic resonance imaging findings have been published in the literature before. Sonoelastography (SE) is an imaging method that allows us to evaluate tissue stiffness in vivo. Many studies have shown that SE increases the specificity of B-mode US in the discrimination of benign-malignant lesions. In this case report, it was shown that PASH is of high stiffness in shear wave elastography evaluation. To our best knowledge, this is the first case presenting SWE findings of PASH in the published literature.

Keywords: Breast; pseudoangiomatous stromal hyperplasia of the breast; shear wave elastography; ultrasonography.
\end{abstract}

\section{INTRODUCTION}

Pseudoangiomatous stromal hyperplasia (PASH) is a benign entity recently defined by Vuitch et al., and it has two subtypes (1). The first is the subgroup, which is more common and reported as a primary lesion component during the histopathological examination. The second subgroup is rarer, mass-forming this subgroup is called tumoral PASH. There are almost PASH 200 cases have been reported in the English literature. PASH is more common in women having hormone replacement therapy (HRT), premenopausal women, and men with gynecomastia (2). Although its etiology has not been fully established, increased progesterone stimulation is blamed for PASH development.
Although PASH is a benign entity, in radiologic evaluation, there is no specific diagnostic feature. Conventional breast imaging methods, including mammography (MG), ultrasonography (US), and magnetic resonance imaging (MRI) findings have been published in the literature before (3). As a result, patients with these lesions are redirected to biopsy for final diagnosis.

Sonoelastography (SE) is an imaging method that allows us to evaluate tissue stiffness in vivo. Many studies have shown that SE increases the specificity of B-mode US in the discrimination of benignmalignant lesions. The first of the SE techniques 
based on different application bases is strain elastography using internal or external compression

stimuli. The second is shear wave elastography (SWE) imaging obtained by the ultrasound device's acoustic radiation force stimulation. Lesion stiffness in SWE is in the form of objective quantitative data measured by the speed of the shear wave obtained from the tissue. An essential advantage of SWE, unlike strain elastography, is that the practitioner's variability is more consistent. Shear wave velocity is measured in elasticity $(\mathrm{m} / \mathrm{s})$ or Young's moduluselasticity $(\mathrm{kPa})$. There is no consensus on the cut-off value for malignant-benign discrimination, and different results such as 50,70 , and $100 \mathrm{kPa}$ have been reported in various studies (4).

In the literature, there is only one case, which shows strain elastography findings of PASH. In this case report, we aimed to present the SWE findings of PASH in two different cases. To our best knowledge, this is the first case presenting SWE findings of PASH in the published literature.

\section{Case-1}

63-year old post-menopausal female patient with no history of breast disease and HRT presented for routine screening MG. As a result of screening $M G$ findings, she was evaluated with the US (ML 6-15$\mathrm{MHz}$ linear transducer LOGIQ S8; GE Healthcare, Milwaukee, WI). In MG and US, a lesion of ovalshaped, sharply demarcated, the hyperechoic halo was detected at the left breast (Figure 1A, B). In the color Doppler US examination, evident vascular coding was not distinguished in the lesion (Figure 1C). SWE has applied also. While the lesion's central

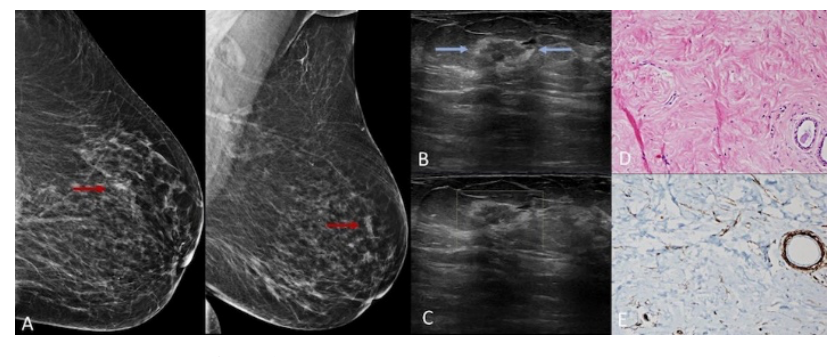

Figure 1. A) Craniocaudal and mediolateral oblique mammography: a well-defined, circumscribed shaped, radiopaque, nodular lesion upper outer quadrant of the left breast (red arrows). B) B-mode ultrasonography: the lesion was detected oval-shaped sharply demarcated, with hyperechoic halo and measured $12 \times 6 \times 7$ $\mathrm{mm}$. (blue arrows). C) Doppler ultrasonography: evident vascular coding was not distinguished. D) Numerous slit-like spaces are present in dense collagenous breast stroma. Unremarkable breast parenchyma is seen at the bottom of the figure (hematoxylin-eosin, original magnification $\times 100)$. E) CD34 shows strong cytoplasmic reactivity in spindle cells (immunoperoxidase, original magnification $\times 200$ )

part was more rigid (red), relatively less stiff areas (blue) in the periphery of the lesion were seen on the colore coded elastography map (Figure 2A). In SWE, the highest elasticity value in the lesion was $8,63 \mathrm{~m} / \mathrm{s}-$ $223,30 \mathrm{kPa}$ (Figure 2B). Due to the absence of the lesion in the patient's previous imaging, the lesion was evaluated as BIRADS 4A, and a core biopsy was performed. The lesion's histopathological diagnosis was reported as tumoral $\mathrm{PASH}$, and the diagnosis was confirmed after excision (Figure 1D, E).

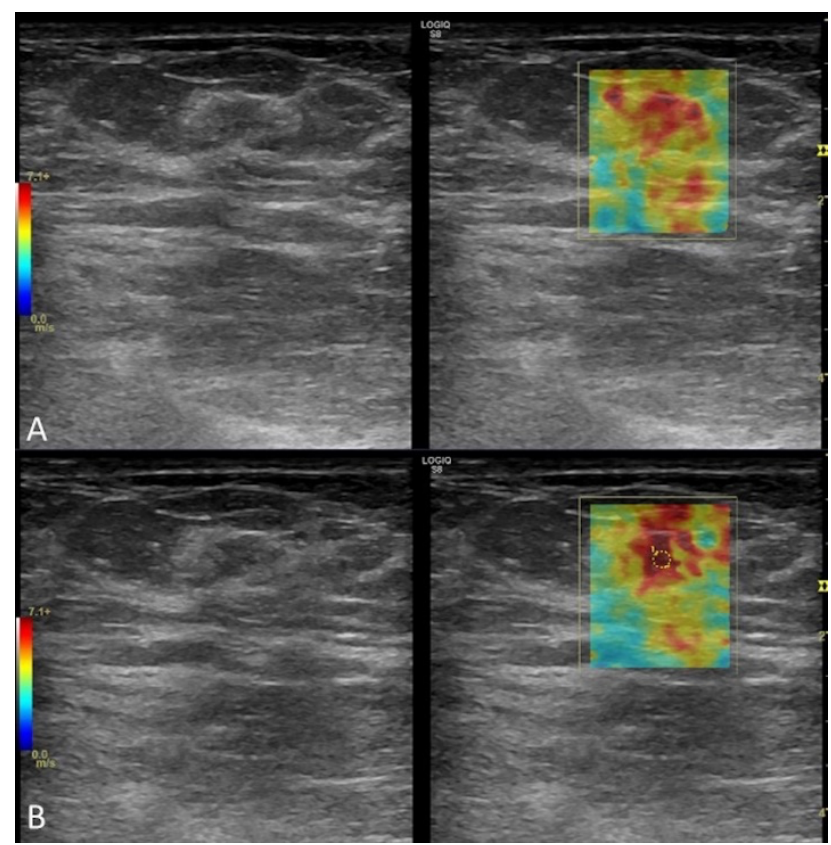

Figure 2. A) While the central part of the lesion was more rigid, relatively less stiff areas in the periphery of the lesion were seen on the color coded elastogram. B) Shear wave elastography: the elasticity score is measured as $8,63 \mathrm{~m} / \mathrm{s}-223,30 \mathrm{kPa}$.

\section{Case -2}

A 20-year-old woman presented with a painless mass complaint in her left breast. US examination performed with $\mathrm{ML}$ 6-15-MHz linear transducer (LOGIQ S8; GE Healthcare, Milwaukee, WI). The US showed a sharp-bound, hypoechoic, homogeneous, oval-shaped lesion in this area (Figure 3A). Colour Doppler US examination revealed vascularity in the lesion (Figure 3B). On the color coded elastography map, the surrounding of the lesion was softer (blue), and the center was relatively harder (red) (Figure 4A). In SWE the highest elasticity value in the lesion was $8,04 \mathrm{~m} / \mathrm{s}-193,89 \mathrm{kPa}$ (Figure 4B). The lesion was evaluated as BIRADS 4A 


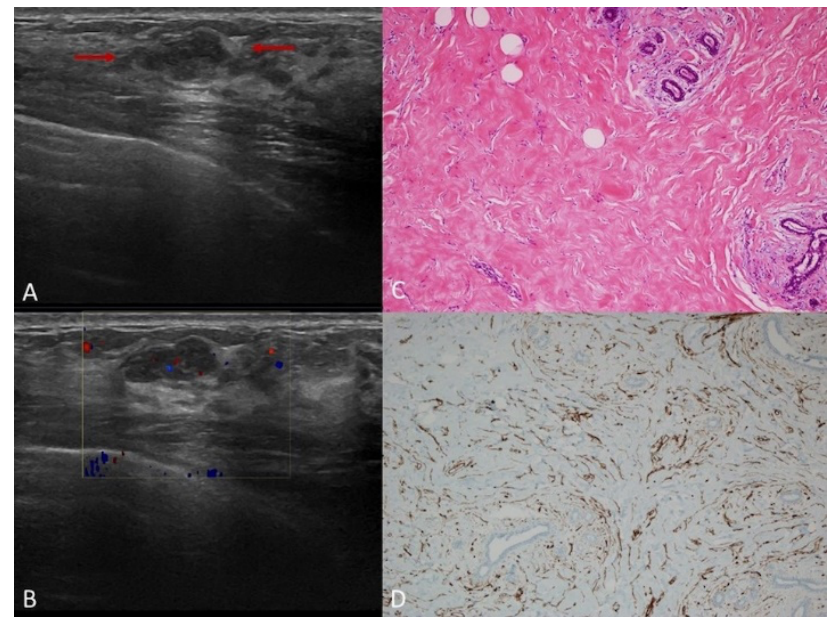

Figure 3. A) B-mode US ultrasonography showed a sharp-bound, hypoechoic, homogeneous, oval-shaped lesion in the left breast (red arrows). B) Colour Doppler US examination revealed vascularity in the lesion. C) Photomicrograph shows typical pseudoangiomatous stromal hyperplasia: anastomosing slit-like empty spaces lined by flattened myofibroblasts without RBCs $(\mathrm{H}$ and E). D) CD34 shows diffuse and strong cytoplasmic reactivity in spindle cells (immunoperoxidase, original magnification x200).

and excised, and histopathological diagnosis was reported as tumoral PASH.

\section{DISCUSSION}

PASH is a diagnosis classified as a benign lesion of mesenchymal origin and reported as a small number of case reports in the literature. In terms of radiological imaging findings, fibroadenoma, hamartoma, and phyllodes tumor should be considered first in the differential diagnosis of $\mathrm{PASH}$. The lesions are usually diagnosed histopathologically due to the lack of specific imaging findings of the lesion. PASH is seen in MG as a non-calcified, solitary, well-circumscribed mass. In the B-mode US, it is distinguished as well-defined, hypoechoic, and oval mass. In MRI, PASH is presented with high signal slit-like spaces on T2-weighted and short tau inversion recovery images. Also, progressive (type 1) enhancement is an important imaging finding for PASH in a dynamic sequence.

SE findings for $\mathrm{PASH}$ are rather limited. In the literature, strain elastography findings of PASH were recognized in only one case (5). The findings of SWE, which we performed in both cases, have not been reported in the literature yet, and are useful in this respect. Histopathologically proven tumoral PASH lesions in both patients were observed in SWE at high stiffness levels. Although this situation led us towards malignant diagnoses in the first plan, considering
PASH's histopathological features, it was understood that SWE findings were compatible with $\mathrm{PASH}$.

In histological examination, the presence of open cleft-like cavities in the dense collagenous stroma is valuable for PASH diagnosis. The spaces are covered with a spindle-shaped layer of the myofibroblast. We think that, due to the increasing number of myofibroblasts, measurements of density increase in tissue and high hardness levels were detected in SWE.

$\mathrm{PASH}$ is a rare benign diagnosis. There is no prominent imaging finding in the radiologic evaluation, and is commonly diagnosed as a possible malignant lesion. It is known that SWE is a useful tool to distinguish benign and malignant lesions. For patients with PASH, adding SWE may contribute to the radiologic diagnosis.

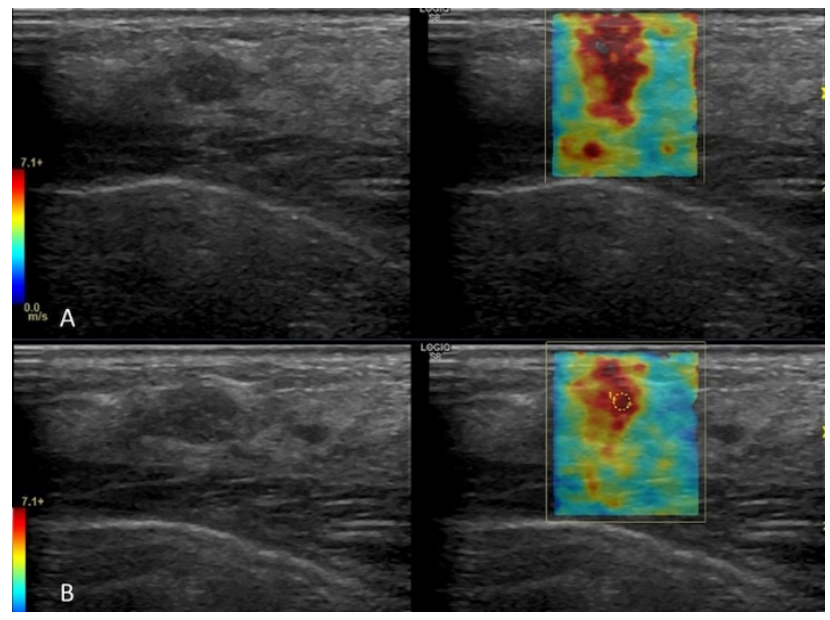

Figure 4. A) On the color coded elastogram the lesion's central part was more rigid; relatively less stiff areas in the periphery of the lesion were seen. B) Shear wave elastography: the elasticity score is measured as $8,04 \mathrm{~m} / \mathrm{s}-193,89 \mathrm{kPa}$.

Conflict of Interest: The authors declare that there is no conflict of interest.

Peer-review: Externally peer-reviewed.

\section{REFERENCES}

1. Vuitch MF, Rosen PP, Erlandson RA. Pseudoangiomatous hyperplasia of the mammary stroma. Hum Pathol 1986;17:185-191.

2. Gresik CM, Godellas C, Aranha GV, Rajan P, Shoup M. Pseudoangiomatous stromal hyperplasia of the breast: a contemporary approach to its clinical and radiologic features and ideal management. Surgery 2010;148:752-758.

3. Jones $\mathrm{KN}$, Glazebrook KN, Reynolds C. Pseudoangiomatous stromal hyperplasia: 
Imaging findings with pathologic and clinical correlation. AJR 2010;195:1036-1042.

4. Athanasiou A, Tardivon A, Tanter M, Siqal-Zafrani B, Bercoff J, Deffieux T, et al. Breast lesions: quantitative elastography with supersonic shear imaging-preliminary results. Radiology 2010; 256:297-303.

5. Yılmaz E, Güngören FZ, Yılmaz $A$ et al. Pseudoangiomatous stromal hyperplasia of the breast: mammosonography and elastography findings with a histopathological correlation. J Breast Health 2015;11:148-51. 